\section{Themenheft zum Lungenkarzinom}



Das Lungen-

karzinom stellt eine der häufigsten Tumorerkrankungen in den westlichen Industrienationen dar. In Deutschland erkranken jähr-

lich etwa 45.000 Menschen, wobei zunehmend auch Frauen betroffen sind. Bei insgesamt ungünstigen Behandlungsergebnissen und kurzen Überlebenszeiten ist das Lungenkarzinom weiterhin die häufigste zum Tode führende Tumorerkrankung. Aufgrund unterschiedlicher klinischer Verläufe und therapeutischer Konzepte kommt heute die größte Bedeutung einer histologischen Unterteilung in kleinzellige und nichtkleinzellige Lungenkarzinome zu. Zur Behandlung des nichtkleinzelligen Lungenkarzinoms ist heute, wie in den Beiträgen des Leitthemenhefts „Nichtkleinzelliges Lungenkarzinom“ eindrücklich gezeigt, ein interdisziplinäres und stadienadaptiertes Therapievorgehen die Regel. Lesen Sie in Ausgabe 08/2011 der Zeitschrift Der Onkologe mehr zu den Themen:

- Pathophysiologie und Molekulardiagnostik beim nichtkleinzelligen Lungenkarzinom

- Stadieneinteilung des nichtkleinzelligen Lungenkarzinoms

- Multimodale Therapie des lokal-fortgeschrittenen nichtkleinzelligen Lungenkarzinoms

- Lokal ablative nichtchirurgische Verfahren beim nichtkleinzelligen Bronchialkarzinom

Bestellen Sie diese Ausgabe zum Preis von EUR 34,- zzgl. Versandkosten bei: Springer Customer Service Center GmbH Kundenservice Zeitschriften

Tel.: +49 6221-345-4303

leserservice@springer.com

www.DerOnkologe.de
PET/CT-Untersuchung mit einem prädiktiven Wert von über $95 \%$ ist heute die Referenzmethode zum nichtinvasiven Staging. Um eine zerebrale Metastasierung auszuschließen, ist immer eine MRT-Gehirnuntersuchung damit zu kombinieren.

- Die Therapie der Wahl ist die komplette R0-Resektion der Oligometastase und des primären Lungenkarzinoms.

- Patienten mit 1-2 synchronen Hirnmetastasen sollten, sofern nicht weitere Organmanifestationen vorliegen, einer konsequenten Behandlung der Metastasen und des Primärtumors unterzogen werden. Bei 1-2 metachronen Hirnmetastasen sollte ebenfalls eine konsequente Metastasenbehandlung mittels stereotaktischer Bestrahlung und/oder Operation erfolgen. Die Patienten sollten auf die Problematik einer erhöhten Rezidivrate im Gehirn und die Möglichkeit der zusätzlichen Ganzhirnbestrahlung hingewiesen werden.

- Für die Indikation einer adjuvanten Chemotherapie bei kurativ behandeltem oligometastasiertem Lungenkarzinom existiert keine dezidierte Studienlage (Einzelfallentscheidung).

- Die Behandlung von Oligometastasen des NSCLC basiert zumeist auf retrospektiven Studien. Deswegen erfordert die Diagnose und Therapie dieser Erkrankung ein patientenabgestimmtes, interdisziplinäres Vorgehen.

\section{Korrespondenzadresse \\ Prof. Dr. Horia Sirbu \\ Thoraxchirurgische Abteilung \\ Uniklinik Erlangen \\ Krankenhausstraße 12 \\ 91054 Erlangen \\ Email: horia.sirbu@uk-erlangen.de}

Interessenkonflikt: Die korrespondierenden Autoren geben an, dass kein Interessenkonflikt besteht.

\section{Literatur}

[1] Pfannschmidt J, Dienemann H (2010) Surgical treatment of oligometastatic non-small cell lung cancer Lung Cancer 69: 251-258

[2] Luketich JD, Martini N, Ginsberg RJ et al (1995) Successful treatment of solitary extracranial metastases from non-smal cell lung cancer. Ann Thorac Surg 60: 1609-1611
[3] Hellman S, Weichselbaum RR (1995) Oligometastases. J Clin Oncol 13: 8-10

[4] Goekenjan G et al (2010) Prävention, Diagnostik, Therapie und Nachsorge des Lungenkarzinoms Interdisziplinäre S3Leitlinie. Pneumologie 64(Suppl 2): 23-155

[5] Kratochwil C, U Haberkorn, Giesel FL (2010) Einsatz der $\mathrm{PET} / \mathrm{CT}$ zur Diagnostik und Therapiestratifizierung des Bronchialkarzinoms. Radiologe 50: 684-691

[6] Rami-Porta R, Ball D, Crowley J et al (2007) The IASLC lung cancer staging project: proposals for the revision of the T descriptors in the forthcoming (seventh) edition of the TNM classification for lung cancer. J Thorac Oncol 2: 593-602

[7] Martini N, Melamed MR (1975) Multiple primary lung cancers. J Thorac Cardiovasc Surg 70: 606-612

[8] Girard N, Ostrovnaya I, Lau C et al (2009) Genomic and mutational profiling to assess clonal relationships between multiple non-small cell lung cancers. Clin Cancer Res 15: 5184-5190

[9] Girard N, Deshpande C, Lau C et al (2009) Comprehensive histologic assessment helps to differentiate multiple lung primary nonsmall cell carcinomas from metastases. Am J Surg Pathol 33: 1752-1764

[10] Postmus PE, Brambilla E, Chansky K et al (2007) The IASLC lung cancer staging project: proposals for revision of the $M$ descriptors in the forthcoming (seventh) edition of the TNM classification of lung cancer. JThorac Oncol 2: 686-693

[11] Voltolini L, Rapicetta C, Luzzi L et al (2010) Surgical treatment of synchronous multiple lung cancer located in a different lobe or lung: high survival in node-negative subgroup. Eur 」 Cardiothorac Surg 37: 1198-1204

[12] Okunieff P, Petersen AL, Philip A et al (2006) stereotactic body radiation therapy (SBRT) for lung metastases. Acta Oncol 45: 808-817

[13] Norihisa Y, Nagata Y, Takayama K et al (2008) M.Stereotactic body radiotherapy for oligometastatic lung tumors. Int J Radiat Oncol Biol Phys 72: 398-403

[14] Saitoh Y, Fujisawa T, Shiba M et al (1999) Prognostic factors in surgical treatment of solitary brain metastasis after resection of non-small-cell lung cancer. Lung Cancer 24: 99-106

[15] Macchiarini P, Buonaguidi R, Hardin M et al (1991) Results and prognostic factors of surgery in the management of nonsmall cell lung cancer with solitary brain metastasis. Cancer 68: 300-304

[16] Flannery TW, Suntharalingam M, Regine WF et al (2008) Long-term survival in patients with synchronous, solitary brain metastasis from non-small-cell lung cancer treated with radiosurgery. Int J Radiat Oncol Biol Phys 72: 19-23

[17] Andrews DW, Scott CB, Sperduto PW et al (2004) Whole brain radiation therapy with or without stereotactic radiosurgery boost for patients with one to three brain metastases: phase III results of the RTOG 9508 randomised trial. Lancet 363(9422): 1665-1672

[18] Burt M, Heelan RT, Coit D et al (1994) Prospective evaluation of unilateral adrenal masses in patients with operable nonsmallcell lung cancer. Impact of magnetic resonance imaging. J Thorac Cardiovasc Surg 107: 588-589

[19] Feliciotti F, Paganini AM, Guerrieri M et al (2003) Laparoscopic anterior adrenalectomy for the treatment of adrenal metastases. Surg Laparosc Endosc Percutan Tech 13: 328-333

[20] Tanvetyanon T, Robinson LA, Schell MJ et al (2008) Outcomes of adrenalectomy for isolated synchronous versus metachronous adrenal metastases in non-small-cell lung cancer: a systematic review and pooled analysis. J Clin Oncol 26: 1142-1147

[21] Ambrogi V, Tonini G, Mineo TC (2001) Prolonged survival after extracranial metastasectomy from synchronous resectable lung cancer. Ann Surg Oncol 8: 663-666

[22] Lang H, Nussbaum KT, Weimann A, Raab R (1999) Liver resection for noncolorectal, non-neuroendocrine hepatic metastases. Chirurg 70: 439-446

[23] Katz AW, Carey-Sampson M, Muhs AG et al (2007) Hypofractionated stereotactic body radiation therapy (SBRT) for limited hepatic metastases. Int J Radiat Oncol Biol Phys 67: 793-798

[24] Schultheis KH, Schroeder-Finckh R, Schultheis A et al (2008) Radiofrequenzablation von Lungentumoren Eine wirklich effektive Therapiemaßnahme? Chirurg 79: 963-970 